\title{
Hypertension and cardiovascular risk factors when treating cancer patients: underrecognised and undertreated
}

\author{
Hani Essa ${ }^{1}$ Andrew R. Pettitt ${ }^{2} \cdot$ Gregory Y. H. Lip $\mathbb{1}^{1,3}$
}

Received: 2 July 2020 / Revised: 24 July 2020 / Accepted: 5 August 2020 / Published online: 12 August 2020

(c) The Author(s), under exclusive licence to Springer Nature Limited 2020

Cancer and cardiovascular disease (CVD) accounts for nearly $50 \%$ of all mortality in the United States (US) [1]. It is common practice to view cardiovascular (CV) risk factors and cancer as two separate disease processes. However, the vast similarities between both, and multiple interactions, suggest some element of shared underlying biology [2]. The demographic profile of oncology patients shares many similarities with the profile of patients with CVDs, and as a result, cardiac condition often co-exist and are a frequent complicating factor during complex and aggressive cancer treatments [3]. Furthermore, CVD is disproportionately represented in survivors of cancer [4], likely multifactorial secondary to downstream treatment effects and factors that resulted in the cancer initially. In the US, improvements in detection, early diagnosis and treatment of cancer have resulted in a marked reduction of overall mortality in the last 20 years [5]. This pattern is likely to be the same in other countries with developed healthcare systems. This means that the burden of CVD in cancer is likely to increase in line with the increasing survivors of cancer. In oncology, CVD is a leading cause of morbidity and mortality [6]. In 2009, after increasing recognition of this issue, the International Cardio-Oncology Society was born [7]. Cardiooncology is a relatively new clinical field focusing on the diagnosis, prevention and treatment of the CV consequences of cancer and its treatment.

$\triangle$ Gregory Y. H. Lip

gregory.lip@liverpool.ac.uk

1 Liverpool Centre for Cardiovascular Science, Liverpool Heart \& Chest Hospital, University of Liverpool, Liverpool, UK

2 Department of Molecular and Clinical Cancer Medicine, University of Liverpool, and Clatterbridge Cancer Centre NHS Foundation Trust, Liverpool, UK

3 Aalborg Thrombosis Research Unit, Department of Clinical Medicine, Aalborg University, Aalborg, Denmark
The scale of CVD prevalence in oncology cannot be understated. Retrospective data in survivors of childhood cancers demonstrate that these patients have an $800 \%$ increased risk of CVDs [8]. Compared to their cancer free siblings, the relative risk of coronary artery disease and heart failure was over $10 \times$ higher [8]. Similar results are also seen in adult data, in 2019, retrospective data from 108,215 cancer survivors and 523,541 controls were reviewed and CVD risks compared [9]. The authors concluded that survivors of most site-specific cancer had increased long-term risks for CVD compared to the general population; however, there was significant variation between cancer sites [9]. Alarmingly, this pattern is also seen in childhood cancer survivors, for whom CVD was the leading cause of death above the age of 60 [10].

Hypertension is one of the most common CVD risk factors in cancer patients [11]. The aetiology of hypertension in this cohort is complex and multifactorial based on the type of cancer treatment, type of malignancy and intrinsic patient factors such as age and pre-existing comorbidities. Therefore, prevalence is difficult to estimate as it is often context specific and therefore difficult to generalise to all oncology situations. A large variety of antineoplastic agents have been linked to hypertension, none more so as angiogenesis inhibitors. All angiogenesis inhibitors have been linked to hypertension [12]. A 2017 metaanalysis of 77 studies looking at 11 different angiogenesis inhibitors demonstrated an odds ratio of 5.28 (95\% confidence interval (CI) of 4.53-6.15) for new onset hypertension. The issue with angiogenesis inhibitors is so prevalent as to have resulted in a consensus document of expert recommendations by the Cardiovascular Toxicities Panel of the National Cancer Institute regarding the assessment, surveillance and management of hypertension when utilising this class of medications [13]. However, hypertension has been linked to multiple other agents including but not limited to antimetabolites such as gemcitabine, mitotic agents such as vincristine, proteasome 
inhibitors such as carfilzomib and adjuvants such as steroids and non-steroidal anti-inflammatories [14].

Cancer patients are more likely to suffer CV risk factors such as hypertension, diabetes and dyslipidaemia and when these are adjusted for, have higher rates of progression to CVD as compared to the general population [4]. This is in keeping with the biological plausibility that cancer and CVD share some underlying processes. Inflammation is one such factor implicated in both cancer and CVD [15]. Inflammatory pathways have been implicated in the initiation and progression of both neoplasm and atherosclerosis. Prognostically, oncology patients with CV risk factors have worse outcomes compared to their peers [4]. Cigarette smoking, obesity, lack of exercise, alcohol, increasing age, diabetes and diet have all been implicated in causing both CVD and cancer [2]. This highlights the importance of active risk factor management in this cohort, even more so than in the general population.

Given the large body of evidence demonstrating that CVD is more common in patients with cancer, one may wonder if this relationship is bidirectional, i.e. do CVD patients suffer a larger burden of cancer than would be expected. Whilst the data in this area are more sparsely populated, there is strong evidence for a relationship demonstrating that CVD patients are more likely to suffer from cancer. A 2016 prospective study looking at 1081 patients who suffered heart failure after myocardial infarction found that they were at a higher risk of cancer compared to patients who suffer myocardial infarction but do not progress to heart failure (adjusted Hazard ratio 1.92, 95\% CI, 1.11-3.11) [16]. This has also been replicated in data published from the Framingham heart study, this looked at 12,712 participants free from CVD and cancer at baseline. These participants were followed up and the data show that in those who developed subsequent $\mathrm{CV}$ events there was a $>7$-fold higher risk of subsequent cancer [17]. There is also evidence that interventions that focus on reducing $\mathrm{CV}$ risk factors also reduce the risk of incipient cancer [18]. Figure 1 demonstrates the shared CV risk factors inherent to both cancer and CVD.

The overlapping risk factors for CVD and cancer suggest that there is an element of shared underlying aetiology. Chronic inflammation is one such pathway; however, there are likely to be others at play. The knowledge that oncology

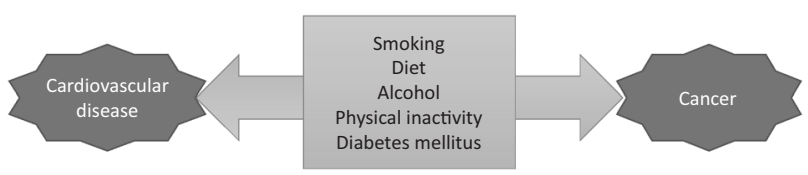

Fig. 1 Theoretical model of bidirectional relationship of cardiovascular disease (CVD) and cancer. There is strong evidence that cancer patients suffer an excess burden of CVD and in turn that CVD patients are more likely to suffer cancer. patients are at a substantially higher risk of $\mathrm{CV}$ risk factors should mean that this cohort undergoes targeted screening to prevent downstream CVD. This is already the case with angiogenesis inhibitors, where a position document by the European Society of Cardiology in 2016 advocated for a $\mathrm{CV}$ risk factor assessment for all patients prior to treatment [19]. Moving forward, this practice should be extended to all cancer patients at all stages of their treatment.

\section{Compliance with ethical standards}

Conflict of interest HE has nothing to declare. GYHL reports consultancy and speaker fees from Bayer, Bayer/Janssen, BMS/Pfizer, Biotronik, Medtronic, Boehringer Ingelheim, Microlife, Roche and Daiichi-Sankyo outside the submitted work. No fees received personally. ARP has received research funding from AstraZeneca, Celgene, Chugai, Gilead, GSK/Novartis, Napp and Roche and speaker fees and hospitality from Celgene, Gilead and Roche. The authors declare that they have no conflict of interest.

Publisher's note Springer Nature remains neutral with regard to jurisdictional claims in published maps and institutional affiliations.

\section{References}

1. Xu J, Murphy SL, Kochanek KD, Bastian BA. Deaths: final data for 2013. Natl Vital Stat Rep. 2016;64:1-119.

2. Koene RJ, Prizment AE, Blaes A, Konety SH. Shared risk factors in cardiovascular disease and cancer. Circulation. 2016;133: 1104-14.

3. Hasin T, Gerber Y, McNallan SM, Weston SA, Kushwaha SS, Nelson TJ, et al. Patients with heart failure have an increased risk of incident cancer. J Am Coll Cardiol. 2013;62:881-6.

4. Armenian SH, Xu L, Ky B, Sun C, Farol LT, Pal SK, et al. Cardiovascular disease among survivors of adult-onset cancer: a community-based retrospective cohort study. J Clin Oncol. 2016;34:1122-30.

5. Siegel RL, Miller KD, Jemal A. Cancer statistics, 2020. CA Cancer J Clin. 2020;70:7-30.

6. Oeffinger KC, Mertens AC, Sklar CA, Kawashima T, Hudson MM, Meadows AT, et al. Chronic health conditions in adult survivors of childhood cancer. N Engl J Med. 2006;355:1572-82.

7. Lenihan DJ, Cardinale D, Cipolla CM. The compelling need for a cardiology and oncology partnership and the birth of the International CardiOncology Society. Prog Cardiovasc Dis. 2010; 53:88-93.

8. Akam-Venkata J, Franco VI, Lipshultz SE. Late cardiotoxicity: issues for childhood cancer survivors. Curr Treat Options Cardiovasc Med. 2016;18:47.

9. Strongman H, Gadd S, Matthews A, Mansfield KE, Stanway S, Lyon AR, et al. Medium and long-term risks of specific cardiovascular diseases in survivors of 20 adult cancers: a populationbased cohort study using multiple linked UK electronic health records databases. Lancet. 2019;394:1041-54.

10. Fidler MM, Reulen RC, Winter DL, Kelly J, Jenkinson HC, Skinner R, et al. Long term cause specific mortality among 34489 five year survivors of childhood cancer in Great Britain: population based cohort study. BMJ. 2016;354:i4351.

11. Piccirillo JF, Tierney RM, Costas I, Grove L, Spitznagel EL. Prognostic importance of comorbidity in a hospital-based cancer registry. JAMA. 2004;291:2441-7. 
12. El-Kenawi AE, El-Remessy AB. Angiogenesis inhibitors in cancer therapy: mechanistic perspective on classification and treatment rationales. Br J Pharm. 2013;170:712-29.

13. Maitland ML, Bakris GL, Black HR, Chen HX, Durand JB, Elliott WJ, et al. Initial assessment, surveillance, and management of blood pressure in patients receiving vascular endothelial growth factor signaling pathway inhibitors. J Natl Cancer Inst. 2010;102:596-604.

14. Cohen JB, Geara AS, Hogan JJ, Townsend RR. Hypertension in cancer patients and survivors: epidemiology, diagnosis, and management. JACC CardioOncol. 2019;1:238-51.

15. Libby $\mathrm{P}$, Kobold S. Inflammation: a common contributor to cancer, aging, and cardiovascular diseases-expanding the concept of cardio-oncology. Cardiovasc Res. 2019;115:824-9.

16. Hasin T, Gerber Y, Weston SA, Jiang R, Killian JM, Manemann $\mathrm{SM}$, et al. Heart failure after myocardial infarction is associated with increased risk of cancer. J Am Coll Cardiol. 2016; 68:265-71.

17. Lau E, Paniagua SM, Liu E, Jovani M, Li S, Takvorian K, et al. Abstract 12269: The Association of Cardiovascular Disease and Future Cancer. Circulation. 2019;140(Suppl_1):A12269-A.

18. Rasmussen-Torvik LJ, Shay CM, Abramson JG, Friedrich CA, Nettleton JA, Prizment AE, et al. Ideal cardiovascular health is inversely associated with incident cancer: the atherosclerosis risk in communities study. Circulation. 2013;127:1270-5.

19. Zamorano JL, Lancellotti P, Rodriguez Muñoz D, Aboyans V, Asteggiano R, Galderisi M, et al. 2016 ESC position paper on cancer treatments and cardiovascular toxicity developed under the auspices of the ESC Committee for Practice Guidelines: the task force for cancer treatments and cardiovascular toxicity of the European Society of Cardiology (ESC). Eur Heart J. 2016;37:2768-801. 